\title{
Kinematics of Two-Roller-Driven Ball for RoboCup Soccer Robot
}

\author{
Shota Chikushi \\ Graduate School of Life Science and Systems Engineering, Kyushu Institute of Technology \\ 2-4 Hibikino, Wakamatsu, Kitakyushu, 808-0196, Japan \\ E-mail: chikushi-shota@edu.brain.kyutech.ac.jp \\ Tharindu Weerakoon \\ Graduate School of Life Science and Systems Engineering, Kyushu Institute of Technology \\ 2-4 Hibikino, Wakamatsu, Kitakyushu, 808-0196, Japan \\ E-mail:weerakoontharindu@brain.kyutech.ac.jp \\ Takashi Sonoda \\ Graduate School of Life Science and Systems Engineering, Kyushu Institute of Technology \\ 2-4 Hibikino, Wakamatsu, Kitakyushu, 808-0196, Japan \\ E-mail: t-sonoda@brain.kyutech.ac.jp \\ Kazuo Ishii \\ Graduate School of Life Science and Systems Engineering, Kyushu Institute of Technology \\ 2-4 Hibikino, Wakamatsu, Kitakyushu, 808-0196, Japan \\ E-mail: ishii@brain.kyutech.ac.jp
}

\begin{abstract}
RoboCup is a competition designed to promote the research related to Artificial Intelligence (AI). We have also organized a RoboCup soccer team "Hibikino-Musashi" and work on one of AI research topics, co-operated behavior control system using multiple autonomous mobile robots. In order to realize co-operated behavior in the soccer game, the research of ball handling system is one of the important issues capable of dribbling with a ball and passing the ball to teammate robots. In this paper, the kinematics of a two-roller-drive ball controller, which are attached in front of the soccer robot and designed to have friction on the upper side of the ball, is proposed. The forward and inverse kinematics between the ball-motion and two active rollers are derived, and the ball control mechanism has been developed and evaluated based on the results of simulations and experiments.
\end{abstract}

Keywords: RoboCup, kinematics of two-roller-drive ball controller, ball control mechanism

\section{Introduction}

The robot competition "RoboCup" is proposed to be a landmark project whose target is to realize the robot soccer team to defeat the human World Cup champion team until 2050. The RoboCup is expected to encourage researchers to develop and promote Artificial Intelligence (AI) and robotics from different aspects [1], and one of whose important concepts is that the progress of AI needs its body, embodiment.

The soccer robots should be autonomous and make a goal in the dynamic environment where many robots move around intricately intertwined with other friend and opponent robots. On the process of developing the co-operated behaviors using soccer robots, the invention of new technologies has been expected because not only 
the technical issues of robot development but also human-robot interactions should be considered, e.g. safety regulations not to damage human and robots themselves, estimation of human will in real-time. In the near future, the robot technology will get closer and inevitable.

We also have organized a RoboCup soccer team "Hibikino-Musashi" for RoboCup Soccer Middle Sized League (MSL) and working on co-operated behavior control system using multiple autonomous mobile robots. To drive intelligence of robots forward, MSL rules are annually reviewed and updated. In recent years, the top teams' robots show collaborated behaviors to make goals and various ball control mechanisms have been developed to dribble with a ball and keep it in good position for reliable pass. The ball control system is one of the important issues to have collaboration between robots. The path plan changes whether robots can keep a ball actively during dribbling or not.

The soccer robot of "Hibikino-Musashi" is shown in Fig. 1. The robot can move omni-directionally using three omni-wheels in the bottom and an omni-vision camera system on the top, and is controlled by a mounted laptop computer. Comparing with our previous robots [2], the robot can move 1.5 times faster and has the ball control device in the front.

In this paper, the kinematics of a two-roller-driven ball controller, which are mounted in the front of the soccer robot and designed to have friction on the upper side of ball, is discussed. The forward and inverse kinematics between the ball motion and two active rollers is derived and evaluated based on the results of simulations and experiments.

\section{Hardware design and concept of a two-roller- driven ball}

Several different ball control mechanisms for soccer robot have been reported in the literatures [3], which are mainly classified into two types. One is designed to control a ball by using two active rollers and another one is by active or passive arms [4]-[5]. The natural selection of soccer robots going on, the former mechanism has become the mainstream. The advantage of the ball controller using active rollers is capable of keeping the ball even in the lateral- and reverse-motion if the rollers are well controlled. On the other hand, the disadvantages of the mechanism are the complexity in design and the ball motion control algorithm suitable to the robot motion is needed. The ball should rotate smoothly with respect to the traveling direction of the robot to satisfy the RoboCup regulation. Regarding its size, the ball control mechanism is restricted not to cover more than one-third of the ball [6]. The RoboCup team Tech United developed the mechanism using two rollers and a solenoid kicker which can keep the ball and select loop-pass or grounder-pass [7]-[9]. The RoboCup team CAMBADA developed using two omnirollers and shows the similar function [10]. The RoboCup team NUBOT developed a control system for the ball handling mechanism [11]. However, the forward and inverse kinematics between the ball motion and the rollers are not well discussed, and the ball motion is controlled by heuristic methods [12]-[13]. We introduce the mathematical model regarding a ball motion and active rollers. The studies on the sphere motion have been done for the mobile robots which utilize the spheres as their wheels [14]-[16]. In the RoboCup soccer, the motion control of a ball is also important, however, the positions of fingers to keep a ball and the kinematics of ball motion and active rollers is not discussed.

The overview of mechanical design of the ball handling device is shown in Fig. 2, and the schematic design is in Fig. 3. In the handling device, active rollers are attached in the lever tips to rotate a ball. The two levers move upward passively when the active rollers rotate to bring the ball toward its body, and the springs and dumpers are connected between the body and the levers to absorb the collision impact of the ball. The small omni-wheels in the bottom are passive rollers to prevent the ball from contacting to the body. If the robot detects the approaching of the ball, the active rollers on the levers start to rotate to bring the ball inside the robot, the friction between the ball and rollers generated by the spring force get the ball inside the robot. Corresponding to the robot's behaviors, the active-roller control starts to synchronize with robot's movements and the directions of the ball rotation. The ball handling device is designed to hold the ball by two active rollers and two passive wheels during dribbling.

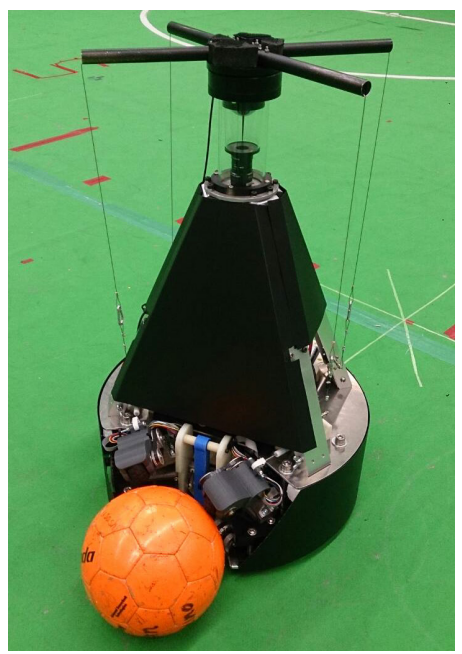

Fig. 1. Soccer robot of "Hibikino-Musashi" 


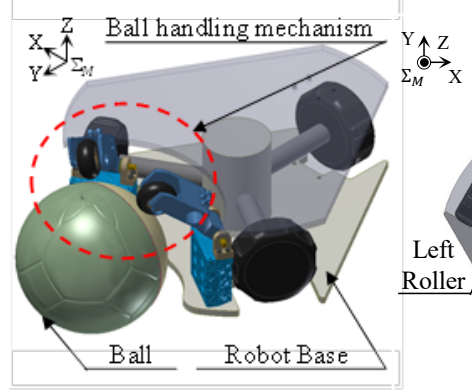

a. Projection view

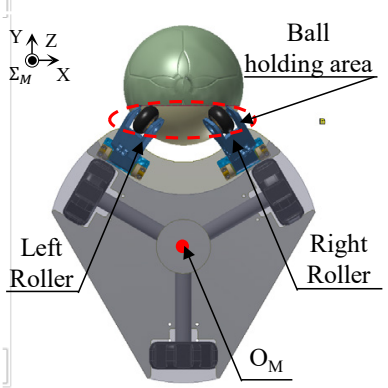

b. Top view
Fig. 2. Overview of the two-roller ball control device

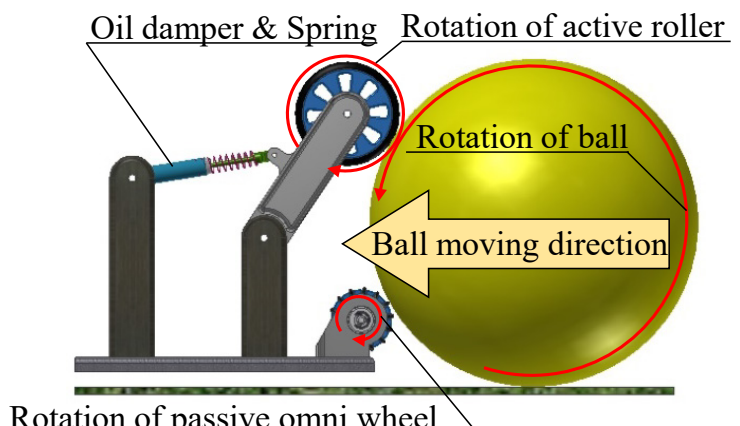

Fig. 3. Concept of the ball handlling device

\section{Kinematics of a two-roller-driven ball}

In this paper, we define that the forward kinematics is the relation from the angular velocities of the two rollers to the ball rotation velocity and the inverse one is from the ball rotation to two rollers rotation. The variables in the equations are defined as shown in the Table 1 , and the graphical representation of the vectors are illustrated in Fig. 4.

\subsection{Inverse Kinematics}

The relationship between the ball velocity vector $\pi \overrightarrow{V_{B}}$ and the active rollers angular velocity vector ${ }^{\Omega} \overrightarrow{\omega_{*}}$ of the can be presented by the inverse kinematics equation given in eq. (1).

$$
\Omega \overrightarrow{\omega_{*}}=f\left({ }^{\pi} \overrightarrow{V_{B}}\right)
$$

While the ball rotates on the flat surface, the ball angular velocity vector $\Omega \overrightarrow{\omega_{B}}$ exists on the plane $\pi$ defined by the three points; the center of the ball $O_{B}$ and the contact points $C_{R}$ and $C_{L}$ [17]-[18]. The normal vector ${ }^{\Omega \perp} \vec{N}$ of the plane $\Omega$ is obtained as in eq. (2). The dot product of normal vector ${ }^{\Omega \perp} \vec{N}$ and ball angular velocity vector ${ }^{\Omega} \overrightarrow{\omega_{B}}$ becomes zero as in eq. (3).

$$
\begin{gathered}
{ }^{\Omega \perp} \vec{N}=\left[\begin{array}{lll}
N_{-} & N_{-} y & N_{-} z
\end{array}\right]^{T}={ }^{\Omega} \overrightarrow{P_{C L}} \times{ }^{\Omega} \overrightarrow{P_{C R}} \\
\Omega \perp \vec{N} \cdot \Omega \overrightarrow{\omega_{B}}=0
\end{gathered}
$$

The ball velocity vector is given by the cross product of the ball angular velocity vector ${ }^{\Omega} \overrightarrow{\omega_{B}}$ and the position vector from the ball center to the ground in eq. (4).

$$
\pi \overrightarrow{V_{B}}=\left[\begin{array}{c}
v_{B_{-} x} \\
v_{B_{-} y} \\
0
\end{array}\right]=\left[\begin{array}{c}
-\omega_{B_{-} y} \cdot r_{B_{-} z} \\
-\omega_{B_{-} x} \cdot r_{B_{-} z} \\
0
\end{array}\right]=\Omega \overrightarrow{\omega_{B}} \times{ }^{\perp \pi} \overrightarrow{r_{B}}
$$

Therefore, the ball angular velocity vector $\Omega \overrightarrow{\omega_{B}}$ is derived as shown in eqs. (5) and (6) using eqs. (3) and (4).

$$
\begin{gathered}
\omega_{B_{-} Z}=\frac{-N_{\_} x\left(-v_{B_{-} y} / r_{B_{Z}}\right)-N_{\_}\left(-v_{B_{-} x} / r_{B_{-} Z}\right)}{N_{\_} Z} \\
\Omega \overrightarrow{\omega_{B}}=\left[\begin{array}{c}
\omega_{B_{-} x} \\
\omega_{B_{-} y} \\
\omega_{B_{-} Z}
\end{array}\right]=\left[\begin{array}{c}
v_{B_{-} y} / r_{B_{-} Z} \\
-v_{B_{-} x} / r_{B_{-} Z} \\
\omega_{B_{-} z}
\end{array}\right]
\end{gathered}
$$

Hence, the roller angular velocity vector $\Omega \overrightarrow{\omega_{*}}$ is expressed as in eq. (7).

$$
\Omega \overrightarrow{\omega_{*}}=\left(\left\|\Omega \overrightarrow{\omega_{B}} \times \Omega \overrightarrow{P_{C *}}\right\| / r_{w}\right)^{\Omega} \overrightarrow{e_{\omega *}}
$$

\subsection{Forward Kinematics}

The forward kinematics from the active rollers angular velocity vector ${ }^{\Omega} \overrightarrow{\omega_{W}}$ to the ball velocity vector ${ }^{\pi} \overrightarrow{V_{B}}$ is represented by eq. (8).

$$
\pi \overrightarrow{V_{B}}=f\left({ }^{\Omega} \overrightarrow{\omega_{*}}\right)
$$

The velocity vector ${ }^{\Omega \perp} \vec{V}_{*}$ at contact point $C_{*}$ is obtained by the cross product of the roller angular velocity vector ${ }^{\Omega} \overrightarrow{\omega_{*}}$ and the position vector ${ }^{\Omega} \overrightarrow{P_{O *}}$ from the roller center $O_{*}$ to the contact point $C_{*}(9)$.

$$
\Omega \perp \vec{V}_{*}=\Omega \overrightarrow{P_{O *}} \times \Omega \overrightarrow{\omega_{*}}
$$

The velocity vector ${ }^{\Omega \perp} \overrightarrow{V_{L}}$ is also expressed by the cross product of the ball angular velocity vector ${ }^{\Omega} \overrightarrow{\omega_{B}}$ and position vector $\Omega \overrightarrow{P_{C *}}$ from ball center $O_{B}$ to contact point $C_{*}$ as in eq. (10).

$$
\begin{aligned}
\Omega \perp \vec{V}_{*}=\left[\begin{array}{l}
v_{*-x} \\
v_{*-} y \\
v_{*_{-} z}
\end{array}\right] & =\Omega \overrightarrow{\omega_{B}} \times \Omega \overrightarrow{P_{C *}} \\
= & {\left[\begin{array}{l}
\omega_{B_{-} y} \cdot P_{*_{-} z}-\omega_{B_{-} z} \cdot P_{*_{-} y} \\
\omega_{B_{-} z} \cdot P_{*_{-} x}-\omega_{B_{-} x} \cdot P_{*_{-} z} \\
\omega_{B_{-} x} \cdot P_{*_{-} y}-\omega_{B_{-} y} \cdot P_{*_{-} x}
\end{array}\right] }
\end{aligned}
$$

Therefore, eqs. (11) and (12) are obtained by eq. (10). 


$$
\begin{array}{r}
\left(\frac{-v_{L_{-} z}+\omega_{B_{-} x} \cdot P_{L_{-} y}}{P_{L_{x}}}\right) P_{R_{-} z}-\left(\frac{v_{L_{-} y}+\omega_{B_{-}} \cdot P_{L_{-} z}}{P_{L_{-}}}\right) \\
\begin{aligned}
& P_{R_{-} y} \\
& =v_{R_{-} x} \\
\left(\frac{-v_{R_{-} z}+\omega_{B_{-} x} \cdot P_{R_{-} y}}{P_{R_{x}}}\right) P_{L_{-} z}-\left(\frac{v_{R_{-} y}+\omega_{B_{-} x} \cdot P_{R_{-} z}}{P_{R_{-} x}}\right) & P_{L_{-} y} \\
= & v_{L_{-} x}
\end{aligned}
\end{array}
$$

The coefficients of $P_{R_{-} y}$ and $P_{L_{-} y}$ in eqs. (11) and (12) are the same and the sum of the two equals to zero as shown in eq.(13).

$$
\left(\frac{v_{L_{-} y}+\omega_{B_{-} x} \cdot P_{L_{-} z}}{P_{L_{-} x}}\right)-\left(\frac{v_{R_{y}}+\omega_{B_{x}} \cdot P_{R_{z}}}{P_{R_{x}}}\right)=0
$$

From the previous equations, the ball angular velocity vector ${ }^{\Omega} \overrightarrow{\omega_{B}}$ can be obtained using eq.(14), and finally, the ball velocity vector ${ }^{\pi} \overrightarrow{V_{B}}$ is obtained as in eq. (15).

$$
\begin{aligned}
\Omega \overrightarrow{\omega_{B}}=\left[\begin{array}{c}
\omega_{B_{-} x} \\
\omega_{B_{-} y} \\
\omega_{B_{-} z}
\end{array}\right]=\left[\begin{array}{c}
\frac{-v_{R_{-} y}+-v_{L_{-} y}}{2 P_{R_{-} z}} \cdot P_{L_{-} y} \\
\frac{-v_{L_{z}}-\omega_{B_{-} x}}{P_{L_{-} x}} \cdot P_{L_{-} z} \\
\frac{-v_{L_{-} x}-\omega_{B_{-}}}{P_{L_{-} y}}
\end{array}\right] \\
\pi \overrightarrow{V_{B}}=\Omega \overrightarrow{\omega_{B}} \times{ }^{\perp \pi} \overrightarrow{r_{B}}
\end{aligned}
$$

Table 1. Variables related to inverse kinematics

\begin{tabular}{cl}
${ }^{\pi} \overrightarrow{V_{B}}$ & Ball velocity vector \\
$\Omega \overrightarrow{\omega_{*}}$ & Roller angular velocity vector \\
${ }^{\perp \Omega} \vec{N}$ & Normal vector of the plane $\Omega$ \\
${ }^{\pi} \overrightarrow{e_{V B}}$ & Unit vector of ${ }^{\pi} \overrightarrow{V_{B}}$ \\
$\Omega \overrightarrow{P_{C *}}$ & Position vector from $O_{B}$ to $C_{*}$ \\
${ }^{\perp} \overrightarrow{r_{B}}$ & Position vector from $O_{B}$ to the ground \\
$\Omega \overrightarrow{\omega_{B}}$ & Angular velocity vector on the plane $\Omega$ \\
$\Omega \overrightarrow{e_{\omega *}}$ & Unit vector of $\Omega \overrightarrow{\omega_{B}}$ \\
${ }^{\perp} \overrightarrow{V_{*}}$ & Velocity vector at the contact point $C_{*}$ \\
${ }^{\Omega} \overrightarrow{P_{O *}}$ & Position vector from $O_{W *}$ to $C_{*}$ \\
$O_{B}$ & Ball center \\
$C_{*}$ & Contact points between the ball and rollers \\
$O_{*}$ & Position vector from $O_{*}$ to $C_{*}$ \\
$r_{w}$ & Roller radius \\
${ }^{*}$ & Index L (Left) or $\mathrm{R}$ (Right) \\
\hline
\end{tabular}

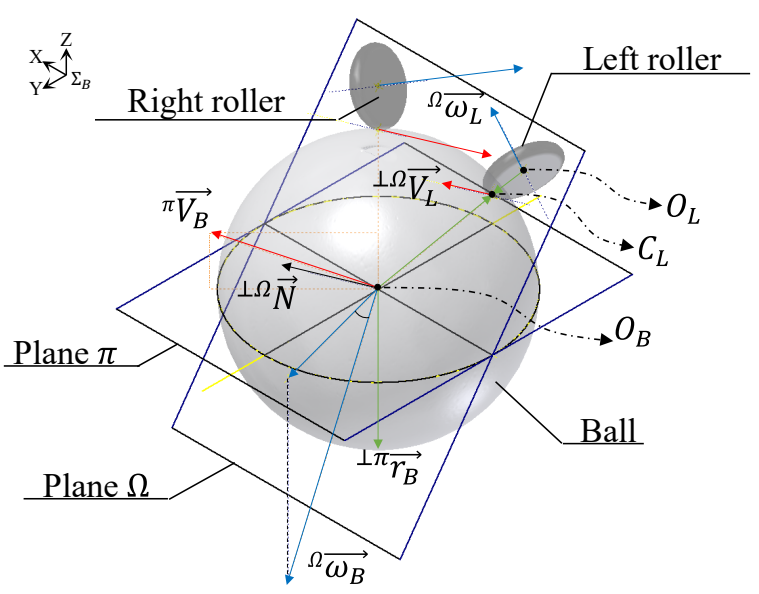

a. Projection view

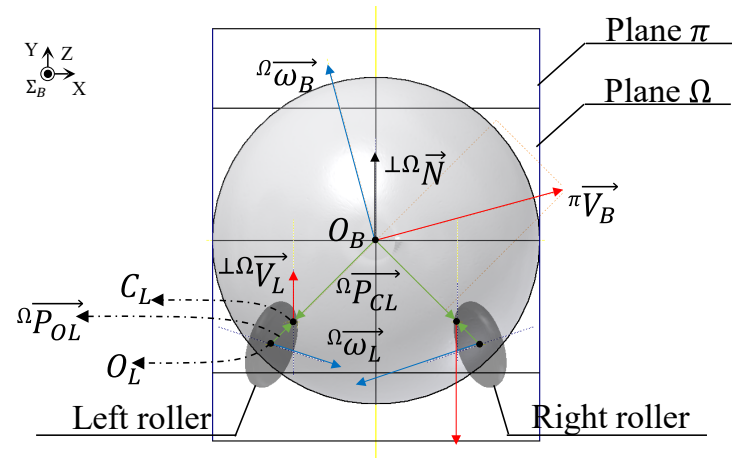

b. Top view

Fig. 4. Vectors in kinematics

\section{Evaluation of the kinematics}

\subsection{Experimental Setup}

To evaluate the kinematics, following experiments are performed to obtain the state variables of ball motion and active rollers. In the experiments, the distance between the roller and the ball is required to be constant, so that an experimental setup has been developed as shown in Fig. 5(a) and (b). The setup is designed so as to change its rollers positions along the ball in arbitrary points and angles. Totally, six encoders are attached to the setup to measure the ball motion and rollers. The four of six encoders are to determine the velocity and the orientation of the setup and the other two encoders are installed on the both omni wheels directly to the active roller motors. Variables used in the experiment are shown in the Table 2. 


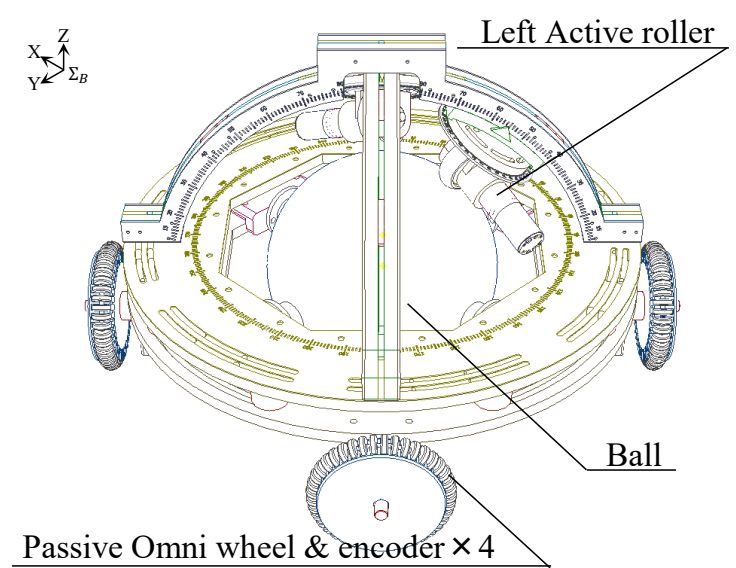

a. Projection view

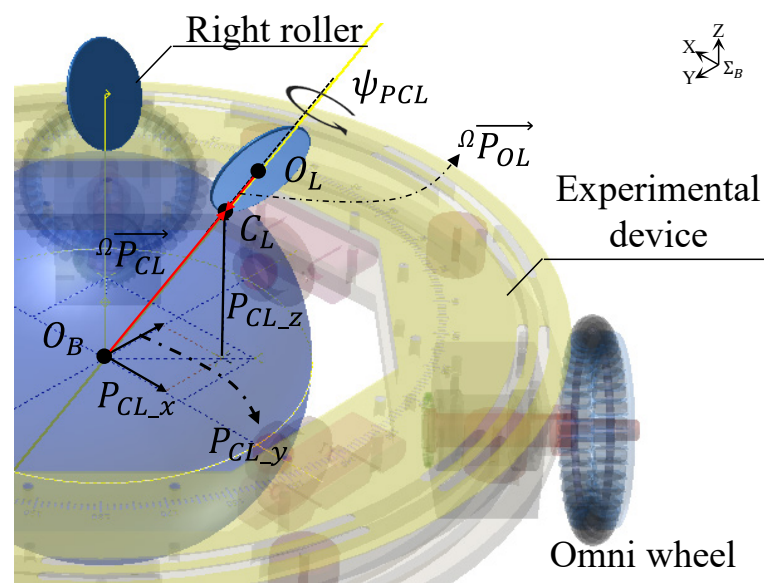

b. Wheel position

Fig. 5. Experimental device of ball handling mechanism

Table 2. Variables used in the experiment

\begin{tabular}{clc}
\hline$r_{B}$ & Ball radius & $0.100 \mathrm{~m}$ \\
$r_{w}$ & Wheel radius & $0.0275 \mathrm{~m}$ \\
$P_{C L_{-} x}$ & x component of ${ }^{\Omega} \overrightarrow{P_{C L}}$ & $0.0400 \mathrm{~m}$ \\
$P_{C L_{-} y}$ & y component of $\Omega \overrightarrow{P_{C L}}$ & $-0.0400 \mathrm{~m}$ \\
$P_{C L_{-} z}$ & $\mathrm{z}$ component of ${ }^{\Omega} \overrightarrow{P_{C L}}$ & $0.0825 \mathrm{~m}$ \\
$\psi_{P C L}$ & Wheel angle around the axis $\Omega \overrightarrow{P_{C L}}$ & $39.5^{\circ}$ \\
\hline
\end{tabular}

\subsection{Experimental Results}

The kinematics between ball motion and rollers is evaluated by translational motions in surge, sway and their combination (see Figs.6-8). The solid and dot lines mean the velocity in $\mathrm{x}$-direction and $\mathrm{y}$-direction, respectively. Black line means the forward kinematics obtained from active wheels' data, and blue are from experimental setup motion.

In the evaluation of surge motion (y-direction in the robot coordinate), the ball is controlled to go backward 270 degree with the velocity of $1.0 \mathrm{~m} / \mathrm{s}$. The comparison of the velocities from setup motion and kinematics calculated by active roller rotation is shown in Fig. 6 . The result shows that the experimental setup motion is well controlled to be $1.0 \mathrm{~m} / \mathrm{s}$, and velocities from the setup and calculation of kinematics become almost same values.

In the sway motion (see Fig. 7), the velocity of $\mathrm{x}$ direction is controlled to be $1.0 \mathrm{~m} / \mathrm{s}$. The experimental data of setup show also good consistent with kinematics calculation. In the experiment of Fig.8, the ball is controlled to go in 225 degree and the velocities in $\mathrm{x}$ and $y$-direction are equal. As the result, the ball moves in about 230 degree, however, the ball motion data and kinematics calculation show good consistent.

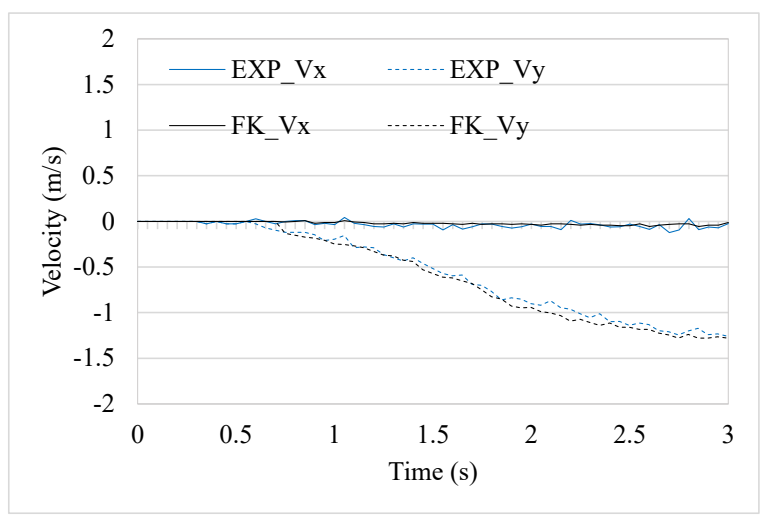

Fig. 6. Experimental results of dribble kinematics $\left(270^{\circ}\right)$

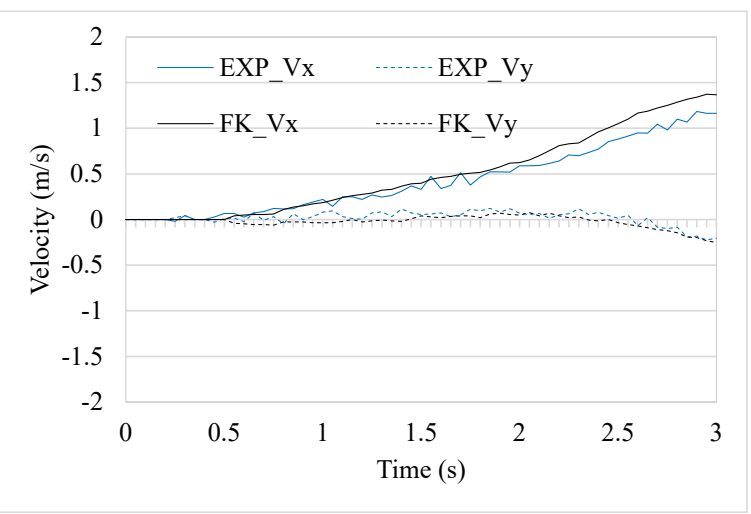

Fig. 7. Experimental results of dribble kinematics $\left(0^{\circ}\right)$ 


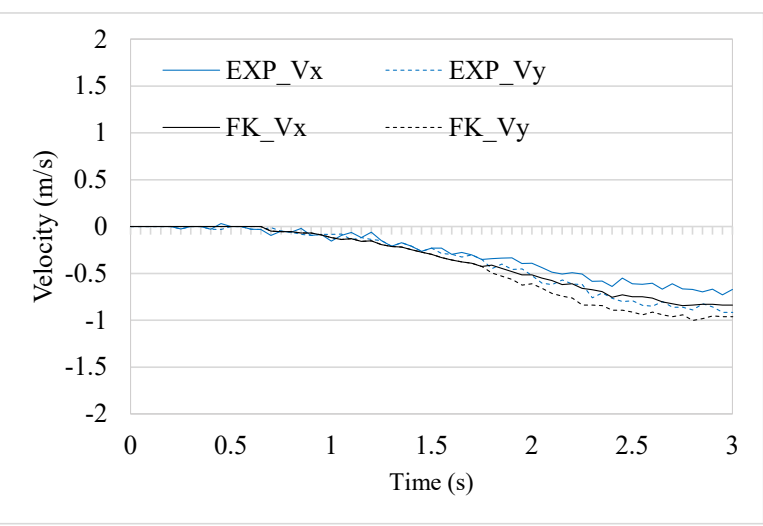

Fig. 8. Experimental results of dribble kinematics $\left(225^{\circ}\right)$

\section{Conclusion}

In this paper, the forward and inverse kinematics between the ball-motion and two active rollers are derived and evaluated by surge and sway motions using developed experimental setup. The results show that the ball motion data and kinematics calculation show good consistent. The ball handling mechanism has enough possibility to realize dribbling behavior of the soccer robots. Using the kinematics of the ball and two-active rollers, the optimal structure depending on robot strategy can be discussed in the future.

\section{References}

1. R. Soetens, R. van de Molengraft, B. Cunha, RoboCup MSL - History, Accomplishments, Current Status and Challenges Ahead, RoboCup 2014: Robot World Cup XVIII. (Springer, 2015), pp624-635

2. S. Chikushi, M. Kuwada, M. Ishikura, et al., HibikinoMusashi Team Description Paper 2016, RoboCup 2016 Leipzig (Leipzig, Saxony, 2016)

3. Stancliff S., Evolution of Active Dribbling Mechanisms in RoboCup, 16-471 Project (2005)

4. K. Fujimoto, K. Ishii, Y. Kitazumi, Development of a ball handling mechanism for a RoboCup Soccer Robot, Robotics and Mechatronics Conference 2011 (Okayama, 2011)

5. T. Hafner, S. Lange, M. Lauer et al., Brainstormers Tribots Team Description, RoboCup 2008 Suzhou (Suzhou, 2008)

6. M. Asada et al., Middle Size Robot League Rules and Regulations for 2015 (2015)
7. J. de Best, R. van de Molengraft, An Active Ball Handling Mechanism for RoboCup, 10th International Conference on Control Automation Robotics \& Vision (ICARCV 2008), (IEEE, Hanoi, 2008), pp. 2060-2065

8. C. Lopez, F. Schoenmakers, G. Naus et al., Tech United Eindhoven Team Description 2014 Middle Size League, RoboCup 2014 João Pessoa (João Pessoa, Paraíba, 2014)

9. C. Lopez, F. Schoenmakers, K. Meessen et al., Tech United Eindhoven Team Description 2016, RoboCup 2016 Leipzig (Leipzig, Saxony, 2016)

10. B. Cunha, A. J. R. Neves, P. Dias et al., CAMBADA'2015: Team Description Paper, RoboCup 2015 Hefei (Hefei, 2015)

11. R. Junkai, X. Chenggang, X. Junhao et al., A Control System for Active Ball Handling in the RoboCup Middle Size League, 2016 Chinese Control and Decision Conference (CCDC), (IEEE, Yinchuan, 2016), pp. 43964402

12. H. Suzuki, T. Yoshida, K. Watanabe et al., RV-Infnity Team Description Paper 2017, RoboCup 2017 Nagoya (Nagoya, 2017)

13. J. de Best, R. van de Molengraft, M. Steinbuch, A Novel Ball Handling Mechanism for the RoboCup Middle Size League, Mechatronics, vol. 21 (Elsevier, 2011), pp. 469-478

14. M. Kumaga, T. Ochiai, Development of a Robot Balanced on a Ball -Application of passive motion to transport-, 2009 IEEE International Conference on Robotics and Automation (ICRA), (IEEE, Kobe, 2009), pp. 4106-4111

15. S. Ishida, H. Miyamoto, Ball wheel drive mechanism for holonomic omnidirectional vehicle, 2010 World Automation Congress (WAC), (IEEE, Kobe, 2010)

16. M. Wada, K. Kato, Kinematic Modeling and Simulation of Active-caster Robotic Drive with a Ball Transmission (ACROBAT-S), 2016 IEEE/RSJ International Conference on Intelligent Robots and Systems, (IROS), (IEEE, Daejeon, 2016), pp. 4455-4460

17. S. Chikushi, T. Weerakoon, K. Ishii et al., Motion Analysis and Control of the Ball Operation for Dribbling Action in RoboCup Soccer Robot, in Proc. 8th International Conference on Soft Computing and Intelligent Systems and 17th International Symposium on Advanced Intelligent Systems (SCIS\&ISIS), (IEEE, Hokkaido, 2016), pp. 532-536

18. K. Kimura, K. Ishii, Y. Takemura, et al., Mathematical Modeling and motion analysis of the wheel based ball retaining mechanism, in Proc. 8th International Conference on Soft Computing and Intelligent Systems and 17th International Symposium on Advanced Intelligent Systems (SCIS\&ISIS), (IEEE, Hokkaido, 2016), pp. 518-523 